\title{
On Chinese Grammar in the Perspective of History - A Review of History of Chinese Grammar by Wang Li
}

\author{
Luo Wanjun, Huang Zengshou \\ College of Liberal Arts, Jiangxi Normal University, Nanchang, China \\ Email address: \\ 1058551150@qq.com (Luo Wanjun),304726484@qq.com (Huang Zengshou)
}

\section{To cite this article:}

Luo Wanjun, Huang Zengshou. On Chinese Grammar in the Perspective of History — A Review of History of Chinese Grammar by Wang Li. Science Innovation. Vol. 8, No. 3, 2020, pp. 76-79. doi: 10.11648/j.si.20200803.15

Received: April 18, 2020; Accepted: June 4, 2020; Published: June 9, 2020

\begin{abstract}
Wang Li pioneered the study of history of Chinese grammar. History of Chinese Grammar by him is the first general history of Chinese grammar in China. History of Chinese Grammar has made a comprehensive and detailed description and analysis of the problems such as the attribution of parts of speech, the usage, meaning and evolution of words, the emergence and development of syntactical structure, word-formation, and so on. Moreover, the analysis of the historical evolution of Chinese reflects the ideas and methods of grammaticalization theory in modern linguistics, which pays attention to observe the critical environment and the syntactic markers of grammatical evolution. The historical perspective of this book and the view of systematicness and graduality needed in the study of grammar also have a profound impact on later researchers. Limited to the level of research at that time, this book inevitably has some views that need to be further discussed and revised, but the review of it is beneficial to the in-depth study of Chinese historical grammar.
\end{abstract}

Keywords: History of Chinese Grammar, Wang Li, Significance, History of Chinese, Advantages and Shortages

\section{在“史”的视野下考察汉语语法一一王力《汉语语法史》述评}

罗婉君, 黄增寿

江西师范大学文学院, 南昌, 中国

邮箱

1058551150@qq.com（罗婉君），304726484@qq.com（黄增寿）

摘要: 王力先生开启了汉语语法史的研究, 其著作《汉语语法史》是中国第一部汉语语法通史。《汉语语法史》对汉 语词类归属、词的用法、意义及其产生和演变、句式的产生和发展、构词法等问题做出了全面而细致的描写和分析, 呈现了汉语语法史的基本面貌, 构成了汉语历史语法研究的基本方面。而且, 对汉语历史演变的分析体现出现代语言 学的语法化理论的思路和方法, 注重寻找语法演变的临界环境和句法标志。本书的历史的视野、语法的系统性和渐变 性观念也对后来的研究者产生了深远的影响。限于当时的研究水平, 本书难免有些需要㽎酌和修正的观点, 但对它的 回顾有益于汉语历史语法的深入研究。

关键词: 《汉语语法史》, 王力, 意义, 汉语史, 得失 


\section{1. 引言}

二十世纪是中国语言学发展的时代, 在中国语言学的 开拓、创新与发展的道路上，王力的工作功不可没。 1936-1937年，王力《中国文法学初探》[1]、《中国文法 中的系词“是”》[2]标志着古汉语语法进入以历史语法描写 研究为主的时期; 1943-1945年， 《中国语法理论》[3] 《中国现代语法》[4]提出的一些思想, 已经包含当代语言 学的倠形; 1958年《汉语史稿》[5]成为中国第一部汉语通 史, 开创了汉语语法史学科, 具有里程碑式意义。这些丰 富的成果无不为《汉语语法史》[6] (以下简称《史》) 的 写作打下扎实的基础。八十年代, 王力在《汉语史稿》的 基础上修正部分观点与例证, 将其中的语法史扩展为 《史》, 于是, 《史》成为中国第一部汉语语法通史。

《史》为汉语语法开创了“史”的研究框架, 以汉语史 的视野对汉语语法的古今变化的基本问题做了回答, 对汉 语语法研究有着框架上的开创意义、方法上的示范作用和 观念上的启示意义。要“前瞻”中国语言学近百年来的发展 脉络, 要“后顾”我们后人还需要做什么, 要探究汉语语法 的演变趋势, 要了解具体的语法现象的演变过程, 就要了 解王力的学术思想, 而《史》是其重要的一本论著。

\section{2. “史”的基本面貌：构建“史”的研究框架}

\section{1. 汉语史研究的基本方面}

《史》以专题的形式，系统地描写了汉语词类、句法 和构词法的历时演变, 使我们能够纵观汉语语法的发展 史。全书将汉语语法分为两大方面: 汉语的词类和构词法。 词类包括名词章、数量和量词章、人称代词章、指示代词 章、疑问代词章、动词章、形容词和副词章、介词和连词 章、系词章、助词章、语气词章, 主要谈及这些词类的主 要成员的用法与意义、产生、更替与发展过程以及实词虚 化的过程和路径, 对词的分类、用法及意义的错误观念, 帮助语法研究回到合理的范围和观念中进行。构词法论述 汉语词的复音化的原因和具体表现; 历代新兴的联绵字、 双音副词及连词的情况。对这两大方面的基本的问题的回 答构成了汉语史研究的基本方面。

\section{2. 对汉语史的独特发现}

句法的历时演变是本书最有独创性的部分。王力从结 构特点发掘汉语的特殊句式，包括判断句、能愿式、连动 式、使成式、处置式、被动式、递系式，探讨了这些句式 的起源与发展、语义特征和功能, 其术语、分析方法及精 妙见解被后人所吸收或运用。目前针对句式开展的研究, 比如连动句、兼语句、是字句、把字句、被动句，是王力 先生及一些老前辈开创的路子。[7]例如, 有关把字句的研 究可追溯到黎锦熙 $[8]$ 的“提宾说”。王力在《中国现代语法》 中称之为“处置式”, 到了 《史》, 改动不大, 坚持从语义 特征方面分析汉语这种特殊句式, 认为“处置式就是 “把” 字句”, 处置式比一般的叙事式语意要重些, 主要作用是 “表示一种有目的的行为, 一种处置”。王力的观点引起众 多学者的讨论。吕叔湘[9]针对把字句的否定语位置、不变
更宾语的动词仍然能进入把字句、“意外的遭遇”也能进入 把字句等问题做了分析。直到现在, 运用构式理论、类型 学视野去研究把字句, 仍然离不开对《史》的观点的回顾。

《史》以史的视野和发展的眼光考察汉语词类、句法 和构词法的历时演变, 展现了汉语语法的发展历史的基本 面貌, 揭示了汉语语法的一些重要特点, 构筑起汉语语法 史研究的大体框架, 后人的汉语史研究、汉语语法研究莫 不直接或间接地受益于书中的精妙见解、分析路子和所用 的文献材料。

\section{3. “史”的研究思路：研究范式的示范作用}

\section{1. 汉语史研究的基本思路}

《史》从动态历时的角度描写了汉语语法现象的历时 演变, 为我们进行了历时语法研究提供了一套可行的思路 和方法。

以“系词的产生及其发展”章为例。首先, 语法的演变 总是在一定的语境中发生, 受制于一定的句法环境。因此 先从句法环境入手, 找出发生演变初始环境。此时, 汉语 的特殊句式往往提供具体的句法环境。“是”字出现在先秦 的判断句中, 充当指示代词, 复指前面的主语。先秦的“是” 并不是系词, 当时判断句的主要格式是“......者, ......也”, 而“是”字出现在判断句的体词性谓语部分, 是“这”的意 思，复指判断句的主语成分，如“富与贵，是人之所欲也”。 然后, 寻找重新分析的契机。此时需要把句法和语义、形 式与意义结合起来考虑。结合句式的结构特点, 对语义进 行分析, 这也是王力的闪光之处。《史》论述, “是”字经 常出现在复杂主语和体词性谓语之间, 渐渐有了将主语和 谓语联结起来的性质，于是“是”的指代义减弱，确认、联 系义加强, 最终变为表判断的语法词。再次, 找到类推的 源动力。类推是语法演变的另一机制, 是语法演变的结果 得以推广的办法。“是”的系词用法从结构复杂的主语类推 到结构简单的主语, 结构简单的主语没有复指的必要, “是”的系词用法完成了它的类推。如“今又倍地而不行仁 政, 是动天下之兵也”的“是”是指代词, 到了“问今世是何 时, 乃不知有汉, 无论魏晋”的“是”是系词。最后, 找出 其演变的条件和形式标志, 以演变条件为标准判断语法现 象的演变情况。“是”字由指示代词演变到系词的条件除了 上述的主语的复杂度减弱之外, 还有同一位置上“此、兹、 斯”等指示代词或“又”等副词的出现。当“此、兹、斯”等指 示代词或“又”等副词出现在“是”的前面时, 就很难将“是” 再解释为“这、那”, 就要考虑它的词性和词义发生了变化。 至于形式标志, 王力在《史》中论述, 系词在判断句中起 经常作用, 系词句在口语里完全代替上古判断句, 是中古 时候的事情。他给出系词“是”成熟的三个标志: “是”字摆 脱判断句句尾“也”字, 单独表判断; 系词“是”字可以受副 词修饰; 系词“是”字前加否定词“不”代替上古的“非”字。 这三大句法标志使他论述的演变条件有可验证性。

《史》的具体分析实质上已经含有语法化的思想和思 路, 富有理论色彩。因此《史》也启示我们, 立足汉语事 实, 开拓出适合汉语的研究路径, 我们才能更好地进行汉 语语法研究。后来学者对系词“是”的形成机制的论证建立 
在王力先生开辟的语法化思路的基础上。梁银峰认为, 系 词 “是”来源于话题化名词短语之后的回指性指示代词 “是”, 它发展为系词的一个关键环节是, “是”所联系的前 后两个名词具有同一性或类属关系, 在这样的句法环境和 语义条件的触发下, 这个回指性指示代词“是”演变为语法 词[10]。

\section{2. 汉语史研究的优良学风}

《史》在真实、典型的传世文献材料的基础上进行描 写工作, 继承了我国语言研究注重实证的优良传统。尤其 是对《论语》、《论衡》、《史记》的辨伪, 体现了《史》 注重甄别、取舍传世文献材料的精神。例如, 黎锦熙认为 判断句的主语是语句时，必须用“是”来复指，所以上古 “是”字是指代词。王力同意黎锦熙的观点, 但对他采用的 例子有不同意见。“是鲁孔丘之徒与？”曰“是也”这个例 子, 王力认为有传抄之误。“是”字是后人加上去的, 实际 上应为“孔丘之徒与? ”, 因为桀溺当面问子路, 不可能说 “那人是鲁孔丘之徒吗?”。有人根据错误的书证来论证 “是”在上古是系词, 就会在其产生与发展问题上发生错 误, 而《史》注重书证的可靠性, 有助于后来的研究者在 扎实的文献基础上展开进一步的研究。语料使用的依据包 括两方面, 一是语料的选择, 口语性和可靠性是基本要求; 二是语料的分析, 有效的例证、剥离口语成分、分析统计 数据以及重视典型语料是基本要求 $[11]$ 。

\section{3. 对现代语言学理论的本土化运用}

《史》尤其注重从句法功能入手分析一些特殊的例 子, 这与当时学界运用美国结构主义描写方法的风潮有 关, 体现了《史》在注重实证的优良传统上借鉴现代语言 学方法的眼光。对一些形式相似的例子, 如果不仔细分析 它出现的句法环境, 就会错误地判断演变发生的时间会所 处的阶段。例如, “其”在上古主要用作定语。有人根据《穀 梁传》中“何以知其是陈君也”一类的句子断定“其”在上古 已有主语用法。《史》指出, 这种用法同主语用法所处的 句法环境很不同，主语没有什么结构上的限制，但这里的 “其”字的句法环境“还不是一个独立的句子”, “只是一个句 子形式（子句）”，相当于“其十名词”，因此它和用作主 语的“其”是很不同的。《史》指出了“其”的特殊句法环境, 从而避免了对代词“其”的演变规律的错误认识。

再看一例。关于现代汉语连动式的研究, 早在中国第 一部语法书《马氏文通》就注意到动词结构连用的现象, 称之为“坐动”。但在《马氏文通》[12]的论述中, “坐动” 内部实际上不止连动式, 还包含兼语式、动词谓语句等, 相互之间的界限比较模糊。黎锦熙《新著国文语法》 [13] 称之为“动词的散动式”, 认为句子中只能有一个主要动 词, 其他动词要么是主要动词的附加语, 要么是主要动词 的补足语, 前者相当于连动式, 后者相当于兼语式, 黎锦 熙将这两种情况统称为“散动式”。实际上, 《马氏文通》 和《新著国文语法》的“坐动”和“散动”说法都是在西方语 法“不定式”理论的基础上提出的，并未关注汉语动词结构 连用的特点。而王力早期著作《中国现代语法》也注意到 这一特殊的语法现象，但把它归为“积累式紧缩句”的一
种, 直到《汉语史稿》也没有专门地描写它。到了《史》, 王力基于汉语事实对连动式进行了具体的描写。他认为这 种句式早在先秦时期就有了, 只是形式比较单一, 主要是 趋向动词和其他动词的连用, 如“王来绍上帝 (尚书·召诰)” 中的“来绍”。晋代以后, 连动式的主要动词不仅可以是前 一个动词, 还可以是后一个动词, 典型代表是“行”字句: “将那氏叔琮, 朱友宁所将军马尽行抽回 (五代史平话·唐 史) ”。“行”相当于词头, 没有什么实义, 动词的实义主 要由“抽回”承担。其次, 晋代以后, 不仅出现了三个动词 连用的形式, 动词间还可以插入宾语, 如“书中吊下金钱 来了也 (元曲·金钱记) ”, 插入了宾语“金钱”, 说明连动 式的发展越发成熟。王力不仅留意到连用格式的扩展, 还 留意到动词之间语义轻重的变化, 这些重要的发现无不立 足于对现代语言学理论的本土化运用。

《史》对历时演变的分析暗含了一套可行的思路和方 法, 富有示范作用; 同时, 针对研究中常见的语料选取与 分析问题, 运用结构主义描写方法去修正它们, 并修正了 学界当时的一些错误观点。这些功夫莫不来自于对汉语语 法研究状况的长期关注, 对注重实证的优良传统的继承及 对国外理论的本土化运用。

\section{4. “史”的语言观: 研究观念的启示意义}

\section{1. 系统的观念}

《史》透露出的语言观能够对我们应该在怎样的“史” 的观念下进行汉语语法演变的考察有所启示。

语法的系统性。语法是一个有机联系的整体，具有很 强的系统性。从宏观上看, 新的语法现象的出现不是偶然 的, 而是受制于当时整个语法系统的状况; 新语法现象的 出现往往是牵一发而动全身, 引起语法系统的一连串变 化。[14]从微观上看, 看一个语法单位的演变, 不能只看 一种句法结构, 而应注意到其他互相联系的句法结构, 综 合全部的句法环境来判定它演变到什么阶段。例如, 唐代, “了”字渐渐虚化为补语, 出现在动词的后面, 仍有“完毕” 义; 同时，还有一种句子的“了”十分像形尾，因为它紧贴 在动词后, 如“今已偿了, 不得久住”。如果孤立地看这种 格式的话, 会认为此时的“了”已有形尾用法, 但是, 如果 把动词后有宾语的格式放在一起看时, 就会知道这时“了” 还不是形尾, 因为它放在宾语后但不紧贴着动词。这种 “了”其实在晋代已经出现, 早于所认为的唐代。真正的形 尾“了”出现在南唐, 因为南唐已出现“了”放在动词后、宾 语前, 如“林花谢了春红”。因此, 要整体地、联系地看待 语法单位出现的所有句法环境, 才能准确地判断语法单位 的性质与所处的演变阶段。

\section{2. 渐变的观念}

《史》还处处渗透着渐变的观念。一个语法化过程通 常是长期的过程, 一个语法化手段的产生和发展受这三个 条件的限制: 语义的适宜性; 特定的句法环境; 较高的使 用频率[15]。因此, 考察一个语法化成分的发展阶段时, 要把它放在史的时间轴上, 观察在它的前后的句法环境有 没有发生临界性的变化, 即使这个变化是细微的。这个观 
念容易接受, 但在实际运用时却容易被忽略。例如, “把” 字的虚化过程有两种句子: 《南史》“即敕把烛看事”; 杜 甫诗“醉把茱英子细看”。这两句的结构形式相似, 且都还 有动词义。如果没有用渐变的眼光看它们, 就看不到其中 的变化。实际上，这两种“把”字后面的句法环境已有所不 同, 句意的重心也逐渐后移。《史》正确地看到了这两种 “把”字之间的差异, 从而正确论述了“把”字的演变过程, 正是因为把握了渐变观。

虽然《史》并没有专门讨论研究观念的问题, 但如果 通读全书, 就会发现其中得真知灼见无不有着语法的系统 观和渐变观的指挥。

当然, 限于当时的研究条件与水平,一些术语已经不 用，如“递系式”、“仂语”、“关系位”，但它背后的语法思 想留了下来。一些观点的印欧语痕迹稍重, 因而有待勘酌, 如“长句的发展”、“五四以后新兴的句法”、“句法的严密 化”。但这些只是小瑕疪, 重要的是《史》为我们展现了 汉语语法演变与发展的基本面貌, 构建了“史”的研究框 架; 动态历时的描写、系统性的分析、扎实的文献功底不 仅为我们示范了汉语语法史的研究思路和方法, 还启示我 们应该在怎样的语言观下开展研究。

\section{5. 结束语}

《史》既开启了汉语历史语法的专书研究, 又为汉语 语法理论体系研究提供重要的启示。的如果想深入研究汉 语语法演变情况及趋势, 《史》仍有丰富的启示; 如果想 了解汉语语法演变的事实、研究的思路和方法, 《史》是 永不过时的入门书。

\section{致谢}

本文是江西省教育厅研究生教改课题《研究生《汉语 语法史》教学改革研究》（编号JXYJG-2015-043）; 江西 省社会科学规划课题《“ $\mathrm{N} 1$ 是 $\mathrm{N} 2$ ”的归类作用及其引申》 （编号14YY15）的阶段性成果之一。

\section{参考文献}

[1] 王力.中国文法学初探[M].北京:商务印书馆,1932.

[2] 王力.中国文法中的系词[J].清华学报,1937(1).

[3] 王力.1944. 中国语法理论 [M]. 济南: 山东教育出版 社,1984.105.

[4] 王力.中国现代语法[M].北京:商务印书馆,1985.86.

[5] 王力.汉语史稿[M].北京: 中华书局,1980.113.

[6] 王力.汉语语法史 [M] .北京: 中华书局.2014.68.115.

[7] 刘铁岩. 论王力先生对现代汉语语法学的贡献(下)[J].河北 师范大学学报.1982(2).

[8] 黎锦熙.新著国语文法[M].北京:商务印书馆,1924.12.44.

[9] 吕叔湘.汉语语法论文集续 [M]. 辽宁：辽宁教育出版 社,2002,400.

[10] 梁银峰.汉语系词“是”的形成机制[].语言研究.2012.第10期 32 卷 (4).

[11] 汪维辉,胡波.汉语史研究中的语料使用问题——兼论系词 “是”发展成熟的年代[J].中国语文.2013（4）.

[12] 马建忠.马氏文通[M].北京: 商务印书馆,1983,34.

[13] 吕叔湘,王海荣. 《马氏文通》读本 $[\mathrm{M}]$.上海: 上海教育出版 社,2005.330.

[14] 石毓智. 语法化的动因与机制 [M]. 北京: 北京大学出版 社,2006,6.

[15] 石毓智,李讷.汉语语法化的历程[M].北京: 北京大学出版 社,2001,6. 